\title{
XCVII. The deflexion by a magnetic field of radium $B$ on recoil from radium $A$
}

\author{
W. Makower M.A. D.Sc. \& E.J. Evans B.Sc.
}

To cite this article: W. Makower M.A. D.Sc. \& E.J. Evans B.Sc. (1910) XCVII. The deflexion by a magnetic field of radium B on recoil from radium A , Philosophical Magazine Series 6, 20:119, 882-886, DOI: $10.1080 / 14786441008636977$

To link to this article: http://dx.doi.org/10.1080/14786441008636977

Published online: 21 Apr 2009.

Submit your article to this journal $\square$

Џ Article views: 2

Q View related articles ๘ 
been worked out by Coffin *, and it was estimated that on this account the effective length of the plates in our experiments was about four per cent greater than their actual length. In the calculation the value 9.8 millimetres has therefore been taken as the length of the plates instead of their real length, $9 \cdot 4$ millimetres, and the numbers given in column 3 of Table II. were thus obtained.

An inspection of the calculated and experimental deflexions shows them to be of the same order of magnitude, and we may therefore conclude that if radium $B$ carries the unit charge of electricity, its atomic weight is of the order to be expected on the disintegration theory of radioactivity.

Our thanks are due to Professor Rutberford not only for supplying us with the radium emanation necessary for the experiments, but also for bis interest in the work.

XCVII. The Deflexion by a Magnetic Fïeld of Radium $B$ on Recoil from Radium $A . \quad B y$ W. Makower, M.A., D.Sc., and E. J. Evans, B.Sc. $\dagger$

\section{[Plate XVIII.]}

T $T$ has been shown by Russ and Makower $\ddagger$ that radium $B$ 1 is positively charged when it recoils from radium $A$ and that the "recoil-atoms" can be deflected by an electric field. Some experiments have lately been made to measure the deflexion suffered by the radium $B$ when it passes through a strong magnetic field. For this purpose a powerful electromagnet was constructed $\S$ capable of giving 10,000 lines per square centimetre over an area $9 \mathrm{~cm}$. by $5 \mathrm{~cm}$., with the poles $2 \mathrm{~cm}$. apart. A glass tube of $2 \mathrm{~cm}$. external diameter

Fig. 1.

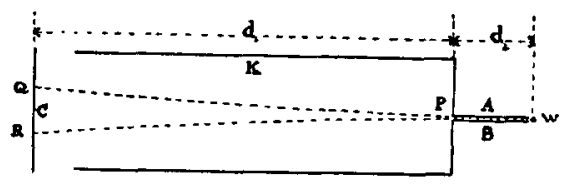

containing the apparatus shown in fig. 1 was placed in this gap between the poles; the glass vessel could be rapidly

* Coffin, Proceedings American Academy, xxxix. No. 19, 1903.

$\dagger$ Communicated by the Authors.

$\ddagger$ Russ and Makower, Phil. Mag. supra, p. 875.

We are indebted to Dr. R. Beattie for designing and supervising the construction of this magnet. 
evacuated when required. The wire $W$, of diameter $0.5 \mathrm{~mm}$., coated with radium $A$ by exposure to the emanation for ten minutes, was placed at the end of the aperture between the two metal plates A, B, $1 \mathrm{~cm}$. long and $0.5 \mathrm{~mm}$. apart. The "recoil-stream" from $W$ passed through this aperture and fell upon the plate $\mathrm{C}$. The metal tube $\mathbf{K}$ served to prevent disturbances by stray electric charges on the glass of the containing vessel. In its path of $7 \cdot 1 \mathrm{cms}$. from $W$ to $C$, the recoil-stream was exposed to a uniform magnetic field.

To carry out an experiment the active wire $W$ was mounted in the position shown in fig. 1, the glass tube was evacuated as quickly as possible, the magnetic field applied and the recoil-stream from $W$ allowed to pass between the plates $A$ and $B$ and fall upon the metal strip C. The distribution of the activity on the plate $C$ was subsequently measured in exactly the same manner as in the experiments on the electrostatic deflexion by means of an a-ray electroscope. To obtain the magnitude of the deflexion suffered by the radium $B$ while passing through the magnetic field, two experiments were performed, one as described and a second one with the field reversed. The distribution of activity over the plate in these two experiments is shown in Pl. XVIII. fig. 2 , curves I. and II. respectively. Now it had been shown by other experiments that the strip $C$ and wire $W$ could be removed and replaced very nearly in the same pusition, so that the distance between the positions of maximum activity in the two experiments just described, gives twice the deflexion suffered by the recoil-stream in each experiment. It will be seen from fig. 2 that the distance between the two maxima is $\cdot 645 \mathrm{~cm}$. The paths of the recoil-streams are circles and the positions of maximum activity $Q$ and $R$ on the strip $C$ are due to matter projected from $W$ describing circular paths passing through $W P Q$ and $W R P$ respectively. If $\mathrm{PC}=d_{1}$ and $\mathrm{WP}=d_{2}$, then if $Q R=d$ and $\rho$ is the radius of curvature of the path of the rays, we have that

$$
d \rho=d_{1}\left(d_{1}+d_{2}\right) .
$$

Since $d_{1}=6.1 \mathrm{~cm}$. and $d_{2}=1 \mathrm{~cm}$., it follows that

$$
\rho=67 \cdot 2 \mathrm{~cm} \text {. }
$$

Since for the experiments described the strength of the magnetic field was 10,800 gauss we have, with the ordinary notation, that

$$
\frac{m v}{e}=\mathrm{H}_{\rho}=7.26 \times 10^{5}
$$




\section{Dr. Makower and Mr. Evans : Deflexion by Magnetic}

The method of finding $\mathrm{H}_{\rho}$ just described is, however, open to certain objections, for it is necessary to make two separate experiments with a direct and reversed magnetic field in order to obtain the deflexion suffered by the recoil-streams. A further disadvantage is that a somewhat large aperture of width, $0.5 \mathrm{~mm}$., was used. Some other experiments were therefore made as follows with a narrower slit and finer wire. The apparatus used is shown in fig. 3 .

Fig. 3.

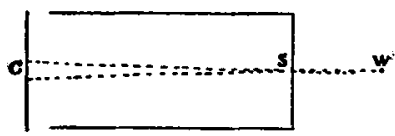

An active wire $W$ of diameter $0.3 \mathrm{~mm}$. was mounted as shown in the figure, $1.25 \mathrm{~cm}$. from the slit $\mathrm{S}$ which was $3.6 \mathrm{~cm}$. from the strip $\mathrm{C}$ placed to receive the recoil-atoms. The whole apparatus was enclosed as before in a glass vessel which could be quickly evacuated and placed between the poles of the electromagnet. The wire $W$ was fixed in position, the field applied and the glass vessel quickly evacuated, and the radium $B$ recoiling from the radium $A$ on the wire allowed to fall on the plate for three minutes. The field was then reversed and the recoil allowed to proceed for another seren minutes until the radium $A$ on the wire had decayed to an inappreciable quantity. The strip $C$ was then removed and placed on a photographic plate in the dark. The radium B distributed over the strip would itself have little or no effect on the photographic plate, but as it decayed radium $C$ was produced in situ, and this by reason of the rays given out by it made an impression on the plate which could be developed in the ordinary way. The result of this experiment is shown in fig. 4 (PI. XVIII.). The two bands on the plate are due to the radium $B$ reaching the plate with the direct and reversed magnetic fields respectively. It will be noticed that the bands are of considerable width and their edges not sharp, indicating that the particles of radium $\mathrm{B}$ on reaching the metallic strip are scattered before being stopped. That this should be so was to be expected considering the relatively low velocity with which the particle must travel. In spite of this scattering, however, it is possible to measure the distance between the middles of the two bands with reasonable accuracy by the method of projecting an inage of the pholograph on to a screen by means of a lantern and measuring the magnification thus produced 
in a manner similar to that adopted by Rutherford in his experiment on the magnetic deflexion of the $\alpha$-particles *.

The distance between the bands was in this way found to be $2.86 \mathrm{~mm}$. Since $d_{1}=3 \cdot 6$ and $d_{2}=4.85$ we have as above

$$
\rho=61 \text {, }
$$

and since the field was 10,700 gauss, we have

$$
\frac{m v}{e}=\mathrm{H} \rho=6.52 \times 10^{5} .
$$

This value is certainly more reliable than that obtained by the first method described above, and is in fair agreement with it.

It is of interest to compare the value of $\mathrm{H} \rho$ obtained with that to be theoretically expected. Now the momentum of the recoil-atom of radium $B$ which is produced on the emission of an $\alpha$-particle from radium $A$ must be the same as the momentum of this $\alpha$-particle, since an atom of radium $A$ gives out only one $\alpha$-particle when it is transformed into radium $B$. It therefore follows that if the charge carried by radium $B$ on recoil is the same as that carried by an $a$ particle, the value of $\mathrm{H} \rho$ for the radium $B$ should be the same as for the a-particle from radium $A$. Now it has been shown by Rutherford that the value of $\mathrm{H} \rho$ of this $\alpha$-particle is $3.48 \times 10^{5}$ or nearly half that for the recoil-atom of radium $B$. It therefore appears that the charge carried by the radium $B$ is half that on an $\alpha$-particle, or, in other words, the atom of radium $B$ carries with it the same charge as the hydrogen ion in electrolysis ; for it is known that the $\alpha$-particle is associated with twice that charge $\neq$.

Although the experimental values so far obtained are not of very great accuracy, it is possible to calculate from the electric and magnetic deflexions of radium $B$ the velocity of these particles and the value of $\frac{e}{m}$. Since the charge carried by the particles has been shown to be the same as that carried by the hydrogen ion in electrolysis, a knowledge of the latter quantity gives the atomic weight of radium $B$.

Taking the path of the particles in an electric field as approximately circular, from the experiments of Russ and Makower we have that with a field of 16,250 volts per

* Rutherford, Phil. Mag. Aug. 1906.

$\dagger$ Rutherford, Phil. Mag. Aug. 1906.

† Rutherford and Geiger, Pruc. Roy. Soc. A., vol. Ixxxi. 1908. 
centimetre the radius of curvature was $12.9 \mathrm{~cm}$. Thus, using electromagnetic units,

$$
\frac{m v^{2}}{e}=2 \cdot 1 \times 10^{13}
$$

and taking the result obtained from the photographic method of measuring the magnetic deflexion, we have

$$
\frac{m v}{e}=6.5 \times 10^{5} \text {. }
$$

Hence $v=3 \cdot 23 \times 10^{7}$ centimetres per second and $\frac{e}{m}=49 \cdot 7$.

Now since for the hydrogen ion in electrolysis $\frac{e}{m}=9 \cdot 6 \times 10^{3}$ the result of these experiments gives the value for the atomic weight of radium $B$ as 194 . Considering the difficulty of the experiments this number is in good agreement with the theoretical value 214. Also it will be noticed that the velocity of the particles has very nearly the value $3 \cdot 27 \times 10^{7}$ centimetres per second, calculated on the assumption that the momentum of the recoil-atoms of radium $B$ is equal to that of the $\alpha$-particle causing it to recoil, and that the atomic weight of radium $B$ is 214 .

It is with pleasure that we take this opportunity of thanking Professor Rutherford for the facilities he has afforded us for carrying out these experiments in his laboratory and also for many valuable suggestions during the course of the work.

XCVIII. Groove-Form and Energy Distribution of Diffraction Gratings. By Ađgustus Trowbridae, Professor of Physics, Princeton University, and R. W. Wood, Professor of Experimental Physics, Johns Hopkins University*.

DRACTICALLY no rigorous investigation has ever been. made of the distribution of energy among the spectra of different orders formed by a diffraction grating, as a function of the wave-length of the light and the form of the grooves. The chief obstacle in the way of such a study is the difficulty of obtaining an exact knowledge of the nature of the furrow cut by a diamond point upon a surface of glass or speculum metal. Microscopical examination teaches us very little or nothing in the case of such fine markings, and it is not safe to infer that the groove will conform at all to the ruling point.

* Communicated bv the Authors. 
FIG. 2.

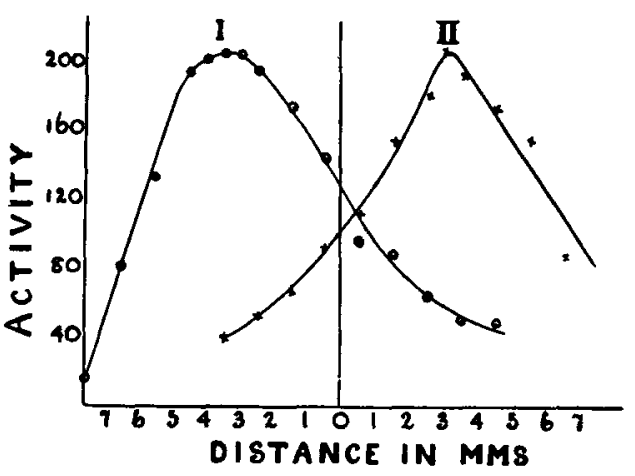

E1G. 4. 